\title{
Low-Order Finite Element Solver with Small Matrix-Matrix Multiplication Accelerated by AI-Specific Hardware for Crustal Deformation Computation
}

\author{
Takuma Yamaguchi \\ The University of Tokyo \\ Tokyo, Japan \\ yamaguchi@eri.u-tokyo.ac.jp
Akira Naruse
NVIDIA corporation
Tokyo, Japan
anaruse@nvidia.com
Tjerk P. Straatsma
Oak Ridge National Laboratory
Knoxville, Tennessee
straatsmatp@ornl.gov

\author{
Kohei Fujita \\ The University of Tokyo \\ Tokyo, Japan \\ fujita@eri.u-tokyo.ac.jp \\ Jack C. Wells \\ Oak Ridge National Laboratory \\ Knoxville, Tennessee \\ wellsjc@ornl.gov \\ Muneo Hori \\ Japan Agency for Marine-Earth \\ Science and Technology \\ Kanagawa, Japan \\ horimune@jamstec.go.jp
Naonori Ueda
RIKEN
Kobe, Japan \\ naonori.ueda@riken.jp
}

\author{
Tsuyoshi Ichimura \\ The University of Tokyo \\ Tokyo, Japan \\ ichimura@eri.u-tokyo.ac.jp \\ Christopher J. Zimmer \\ Oak Ridge National Laboratory \\ Knoxville, Tennessee \\ zimmercj@ornl.gov \\ Lalith Maddegedara \\ The University of Tokyo \\ Tokyo, Japan \\ lalith@eri.u-tokyo.ac.jp
}

\begin{abstract}
This study proposes a fast low-order finite element solver for crustal deformation computations by applying Tensor Core, AI-specific hardware on a Volta GPU. Tensor Core can compute large matrixmatrix multiplications rapidly in half precision. We redesign a state-of-the-art solver algorithm so that lower-precision data types can be used and memory access costs can be reduced even when we use small matrices. With the proposed solver, we solved 13 billion degrees-of-freedom two-layered problems that mimicked the Earth's crust and mantle using 36 compute nodes of Summit. In the matrix-vector kernel, we obtained a 4.1-fold speedup over a standard kernel in a single-precision format. Our proposed solver increased the FLOP count of the entire solver; however, we reduced the time-to-solution by 1.7 -fold since the Tensor Core provided a high effective performance.
\end{abstract}

\section{CCS CONCEPTS}

- Computing methodologies $\rightarrow$ Massively parallel algorithms; - Applied computing $\rightarrow$ Earth and atmospheric sciences.

This work is licensed under a Creative Commons Attribution International 4.0 License.

PASC '20, fune 29-fuly 1, 2020, Geneva, Switzerland

(C) 2020 Copyright held by the owner/author(s).

ACM ISBN 978-1-4503-7993-9/20/06.

https://doi.org/10.1145/3394277.3401860

\section{KEYWORDS}

Transprecision computing, GPU computation, Finite element analysis, Conjugate gradient method

ACM Reference Format:

Takuma Yamaguchi, Kohei Fujita, Tsuyoshi Ichimura, Akira Naruse, Jack C. Wells, Christopher J. Zimmer, Tjerk P. Straatsma, Muneo Hori, Lalith Maddegedara, and Naonori Ueda. 2020. Low-Order Finite Element Solver with Small Matrix-Matrix Multiplication Accelerated by AI-Specific Hardware for Crustal Deformation Computation. In Proceedings of the Platform for Advanced Scientific Computing Conference (PASC '20), June 29July 1, 2020, Geneva, Switzerland. ACM, New York, NY, USA, 11 pages. https://doi.org/10.1145/3394277.3401860

\section{INTRODUCTION}

\subsection{Background of Crustal Deformation Computation}

A better understanding of earthquakes and their generation processes is important for future disaster mitigation. References [32] discuss inverse analysis for the distribution of fault slip. This inversion connects to evaluation of long-term plate movements and future earthquake occurrence. It requires many Green's functions and GPS-based crustal deformation observation data to compare. Green's functions are the responses including post-earthquake deformation for given inter-plate slips, each of which is computed by forward analysis of crustal deformation computation. Crustal deformation observation data has been accumulated as observation systems have been developed (e.g., the GNSS Earth Observation Network System (GEONET) [35] in the area around Japan). Jointly 
with observation networks, inverse analyses with a lot of crustal deformation computations will enhance the capability of monitoring and evaluating earthquake hazard.

To realize the inversion, we need to analyze the time history of the visco-elasto-plastic response of the 3D heterogeneous crust and mantle because of the slip on the plate boundary and faults. Previously, analytic solutions ( [36] for elastic analysis and [10] for visco-elastic analysis) were widely used under the conditions that target domains were homogeneous or horizontally-layered. As crustal structure data has been accumulated [43], [6], [17], models of fault interactions considering fault friction with high resolution have been available. Therefore, the demand for crustal deformation computations using these high-resolution models has increased. Some studies have used low-order finite element modeling (FEM) to accommodate heterogeneity in material properties and complex geometry of the target domain [28], [37], [47]. It is implied that fine spatial resolution in the entire medium surrounding the faults using is necessary, given the heterogeneous nature of the Earth [30], [18]. In addition, FEM has the advantage to satisfy the traction-free condition at the ground surface automatically. Following previous studies, we use FEM for the target problem.

On the other hand, inversion using FEM requires massive computation cost. At least a $\mathrm{km}$-scale resolution is required in the simulation, and visco-elasto-plastic loading and response of the faults and surrounding crust must be captured. When we compute for $1000 \mathrm{~km} \times 1000 \mathrm{~km} \times 100 \mathrm{~km}$ domain with approximately $1 \mathrm{~km}$ resolution, $10^{10}$ to $10^{11}$ degrees of freedom (DOF) are necessary in the FEM. The analysis of $10^{3}$ time steps is required to obtain the time-history response. Moreover, introduction of inverse analysis requires $10^{2}$ to $10^{3}$ case forward analyses.

To conduct these simulations within a realistic timeframe, we must reduce the computation cost. The visco-elasto-plastic response analysis can be realized by a small modification of the solver algorithm for the static response analyses of the linear elastic body (see [37] for visco-elastic analysis); hence discussion of computation cost in linear problems works for that in visco-elasto-plastic analysis. In this paper, we focus on the performance in finite element method for elasto-static problems.

\subsection{Background of Finite Element Solver for Crustal Deformation Computation}

The solver part accounts for the largest proportion of the computation cost in the entire analysis; thus, we focus on the acceleration of the solver. Multi-grid solvers are considered suitable for our targeting problems as they can attain good convergency even for larger-scale problems by reducing long wavelength errors efficiently on their coarsened grids.

There are many variants of multi-grid solver algorithms according to the problem characteristics of the crustal deformation problem (e.g., complexity in geometry, heterogeneity of material properties, and targeting resolution). For example, [39] is proposed for solving large-scale problems in global mantle convection using hexahedral elements and adaptive refinement, and [9] is proposed for solving large-scale static problems with tetrahedral elements. Therefore, it is not straightforward to define a single state-of-the-art solver for comparison in a general setting.
In this paper, we define a multi-grid solver [21] as the baseline solver that is robust for solving a wide variety of tetrahedral mesh problems which combines conjugate-gradient methods to improve robustness of the geometric multi-grid method. It is shown to attain high performance and scalability on large-scale CPU- and GPUbased computer systems [21], [20], [22]. In particular, we compare performance with the SC14 solver as it is the most general and robust. As explained in Section 4, most components are common to the SC14 solver and our proposed solver; thus, we can clearly see the factor of the performance improvement in our proposed method. A variant of this solver using algebraic multi-grid (AMG) exists [9], but regeneration of AMG-matrices in each nonlinear iteration of nonlinear problems leads to high cost in GPU environments, and thus we remove this from comparison. Although the solver performance is compared to a single solver algorithm, the basic algorithm concepts of coarsening and the computational components used in the variety of multi-grid solvers are similar (e.g., matrix-vector products used in smoothers); thus, the idea of acceleration of the key component in the solver is expected to be useful for a variety of problem settings.

\subsection{Motivation for Using AI-specific Hardware}

Considering the diversity of hardware, it is expected that algorithms can be developed for specific hardware that are faster than state-ofthe-art algorithms designed for general hardware. Development of deep learning is expanding the demand to compute matrix-matrix multiplication rapidly. Learning is computationally expensive and can sometimes take up to $10^{1}$ hours as noted in [42], in which matrix-matrix multiplication accounts for the largest proportion of the computational cost. To meet this demand, much hardware specialized for matrix-matrix multiplication has been developed. For example, the NVIDIA Volta GPU [34] has Tensor Core, which was designed to accelerate deep learning [29], while the entire GPU is compatible with general scientific computations. Peak FLOPS performance using single or double precision is 7.8 or 15.6 TFLOPS, respectively, whereas the performance of Tensor Core can reach 125 TFLOPS, so significant acceleration can be achieved. A Tensor Processing Unit (TPU) [12] by Google also targets deep learning, and the second-generation TPU has achieved 45 TOPS per chip. This tendency is expected to continue. In fact, chips designed for artificial intelligence (AI) [5], [4] have been announced by many corporations at Hot Chips, which is known as the conference where up-to-date information about newly developed chips is released.

By utilizing these computational resources, it is possible to benefit from the high peak performance of specialized cores. In this paper, we target acceleration of a solver based on multi-grid algorithms. On the other hand, to use this AI-specific hardware for our target application, the following two points must be resolved.

Firstly, such hardware only supports lower-precision data types because the main target application is deep learning. As described in [14], deep learning does not require very high accuracy. Hence, Tensor Core on a Volta GPU and a TPU only support data types with accuracy less than single precision. A common data type used in deep learning, half precision numbers are composed of a 1-bit sign part, a 5-bit exponent part, and a 10-bit fraction part, as defined in IEEE 754 [27]. Exponent bits and fraction bits are truncated from 
single-precision data types, which are composed of a 1-bit sign part, an 8-bit exponent part, and a 23-bit fraction part. Accordingly, halfprecision numbers have smaller dynamic ranges and less accuracy as compared to single- or double-precision. The minimum and maximum positive values that can be represented by half-precision data type are $10^{-8}$ and 65,504 , respectively. This range is too small for general numerical simulations and heedless implementations easily lead to overflow or underflow; therefore, methods that avoid these conditions are essential. Half-precision data types are unable to guarantee accuracy of more than four digits, so the issue of accuracy is critical to convergence of solvers. A TPU uses another data type called bfloat16 [46] for multiplication, but its accuracy is less than that of half precision because it truncates many bits of the fraction part while keeping the same number of exponent bits as in single-precision data types. Hence, it is also a challenge to apply bfloat16 to iterative solvers.

Secondly, memory access costs are a bottleneck to the performance when small matrices are used. General matrix-matrix multiplications are dense computations and easily parallelized; hence, they are suitable for GPU computations. Methods to execute such multiplications on GPUs have been developed with cuBLAS [33] and MAGMA [45] as major libraries, which are tuned mainly for multiplications involving large matrices. They already have functions to use Tensor Core and these libraries are most straightforward ways to use AI-specific hardware. In these matrix-matrix multiplications, the computation and data access costs are of the orders, $O\left(N^{3}\right)$ and $O\left(N^{2}\right)$, respectively, for matrices of size $N$; thus, as the matrix size increases, memory access costs relatively decrease. The aforementioned hardware has achieved good performance for multiplication operations that are common in typical deep learning applications that use matrices with sizes of more than several hundred. However, the sizes of our targeting matrices are only several dozen elements as we are targeting low-order FEM. Smaller matrix-matrix multiplications appear in various applications (e.g., sparse direct solver [49] and tensor contractions [25]). In these computations, data access costs can be a bottleneck that hampers the utilization of the hardware's full potential. A previous study [3] showed that it was difficult for these libraries to attain good performance for small matrix-matrix multiplication, so optimizations of the target matrix sizes were required.

To use hardware dedicated to matrix-matrix multiplication in lower-precision data types, we have the issues noted above.

In this study, we propose a low-order finite element solver with small matrix-matrix multiplications accelerated by AI-specific hardware. We focus especially on Tensor Core on an NVIDIA Tesla V100 GPU. By designing an appropriate multi-grid preconditioner, we obtain the results at double precision while most computations are executed at a lower precision. Also, we introduce methods to reduce data access costs so that access to the device memory or other memories does not limit performance of the matrix-matrix multiplications. We were able to introduce further optimizations of thread parallelization and memory access for our target computations; thus, our implementation achieves better performance than typical libraries at the expense of versatility. Our proposed algorithm increases the number of computations compared to a conventional solver or a state-of-the-art solver, but the time-to-solution is shorter as most computations are matrix-matrix multiplications that can be computed rapidly on Tensor Core.

Many approaches to use transprecision computing in linear solvers have been proposed [7], [15], [40], [8], [16]. Especially, the solver in [16] can obtain the solution in double precision with refinement of the initial solution computed on Tensor Core. This solver targets dense matrix and its matrix size is $10^{3}$; thus our target problem is assumed to be more difficult to attain reasonable performance. Also, an approach that computes matrix-vector multiplication as matrix-matrix multiplication is proposed in [2]. It uses high-order elements; however, our application uses low-order elements. Therefore, the matrix sizes are smaller and pose a greater challenge to good performance.

Considering the development trend of similar hardware, a successful case to use such hardware by incorporating computations of lower precision and reducing memory access costs is expected to have implications for improvements in the performance of other scientific applications. Our proposed solver is an extension of [23]. In this paper, we describe the methodology in detail and the additional performance measurements conducted in a smaller computation environment.

The remainder of this paper is organized as follows. Section 2 describes the solver algorithm that enables matrix-matrix multiplication on Tensor Core, and Section 3 describes performance tunings for small matrix-matrix multiplications. Section 4 discusses the effectiveness of our proposed method via performance measurements made on the supercomputer, Summit. Section 5 presents our conclusions.

\section{SOLVER ALGORITHM TO USE TENSOR CORE}

In the present study, we focus on the finite element method with second-order tetrahedral elements for the static response of a linear elastic body [19]. To calculate a displacement function $u_{i}$ with double precision, the target equation is:

$$
\frac{\mathrm{d}}{\mathrm{d} x_{i}}\left(c_{i j k l}(\mathbf{x}) \frac{\mathrm{d}}{\mathrm{d} x_{k}} u_{l}(\mathbf{x})\right)=0,
$$

Here, $c_{i j k l}$ denotes the stiffness tensor. $\mathbf{x}$ and $\frac{\mathrm{d}}{\mathrm{d} x_{i}}$ denote the spatial coordinate and differentiation with respect to $x_{i}$, respectively. By discretizing Eq. (1), it results in the following equation:

$$
\mathrm{Ku}=\mathbf{f}
$$

where $\mathbf{K}$ is the global stiffness matrix, $\mathbf{u}$ is the displacement vector, and $\mathbf{f}$ is the force vector.

\subsection{Overview of the solver}

We target the acceleration of this equation-based modeling by utilizing AI-specific architecture such as Tensor Core. We convert the algorithm to a highly dense computational one consisting of matrix-matrix multiplications.

To use Tensor Core's operations, we have to reduce the accuracy of the variables in the solver. So, we use an adaptive conjugate gradient-type solver [11], which roughly solves the same target 
equation by employing conjugate gradient solvers in the preconditioner. This solver has the capability of combining various methods into the preconditioner.

Only an approximate solution is required in the preconditioner; therefore, we can combine the use of several methods to gradually solve the approximated equation at low cost. Especially, we can use lower precision numbers in the preconditioner (see [40] for similar approaches). Fig. 1 shows the developed solver algorithm. Here, all matrix-vector multiplications in the solver are computed by an element-by-element method [48] to reduce memory usage. We use a model mapped to a structured mesh $\operatorname{PreCG}^{v}$, linear tetrahedral elements PreCG ${ }^{c}$, and the target second-order tetrahedral elements PreCG. In addition, we used PreCG $G_{\text {part }}$, which solves parts of the problem that are expected to have large differences from the original model PreCG when using the abovementioned preconditioner. Here, PreCG $G_{\text {part }}$ was extracted from PreCG using an AI-based method [22]. Only this part was then solved in the preconditioner. Furthermore, instead of using a standard voxel finite element in $\operatorname{PreCG}^{v}$, we designed an algorithm with a high mapping accuracy of the original problem PreCG together with a high computational density.

\subsection{Mapping of unstructured problem to structured mesh}

For PreCG ${ }^{v}$, we generate a 3D structured grid model $\bar{c}_{i j k l}$ equivalent to an unstructured grid based on $c_{i j k l}$ of (1). Constant material properties are assigned to each structured grid, which has a size of $(d s / 2)^{3}$, where $d s$ is the size of each voxel element. Here, we target domains of arbitrary size. It is desirable to use a solver algorithm that can be applied robustly; therefore, we use conjugate gradient method for PreCG ${ }^{v} .3 \times 3$ block Jacobi preconditioning [41] is used, which requires less memory consumption than other typical preconditioners. Obtaining an accurate solution with $\mathrm{PreCG}$ leads to the reduction of the more costly PreCG ${ }^{c}$ and PreCG, so we target the generation of $\bar{c}_{i j k l}$ with high mapping accuracy and low construction cost. Following the analyses of [24], we set $\bar{c}_{i j k l}$ as the volume average of $c_{i j k l}$ in each structured grid with a size of $\left(d s / 2^{3}\right)$.

Using this structured grid-based setting enables one to obtain low-spatial frequency solutions with considerable accuracy. These solutions are used as the initial solutions in the latter solvers in the preconditioner. While the low-frequency components of the solution are expected to become accurate because of the coarsening based on physical properties, local errors are expected to increase in places where the differences between $c_{i j k l}$ and $\bar{c}_{i j k l}$ are large. Thus, following [22], we estimated the parts of the problem expected to have large errors using AI and solved only these parts using PreCG $G_{\text {part }}$ (see footnote ${ }^{1}$ for details).

The mapping between solvers is conducted in every outer iteration of the adaptive conjugate gradient solver to enable the equation to be solved roughly many times. Thus, almost all of the computational costs of the conjugate gradient solver in the second-order

\footnotetext{
${ }^{1}$ We used a small problem with characteristics similar to that of the target problem to train an artificial neural network (ANN) that estimates the difference between the solutions obtained using $c_{i j k l}$ and those obtained using $\bar{c}_{i j k l}$. The differences in $c_{i j k l}$ and $\bar{c}_{i j k l}$ and the number of nodal connectivity in the mesh were used as input for the ANN. We applied this ANN to the target problem to inference parts to be solved in PreCG $G_{\text {part }}$.
}

tetrahedral mesh in double precision are transferred to the coarsened solvers in low-precision arithmetic. Note that even though coarsening and low-precision arithmetic are used in the preconditioner, the final double-precision results of the solver are not altered from those of the standard solvers.

\subsection{Introduction of Tensor Core operations to matrix-vector kernel}

$\operatorname{PreCG}^{v}$ is a structured grid-based problem and uses uniform basis functions; hence, it is suitable for attaining shorter time-to-solution as its convergency is better than that in unstructured problems. In addition, matrix-vector multiplication in the $\operatorname{PreCG}^{v}$ solver can be rearranged to matrix-matrix multiplication.

Stiffness matrix for ie-th element can be described as follows:

$$
\mathbf{K}_{(i e)}=\int_{-1}^{1} \int_{-1}^{1} \int_{-1}^{1} \mathbf{B}^{T} \mathbf{D}_{(i e)}\left(r_{1}, r_{2}, r_{3}\right) \mathbf{B d e t}|J| \mathrm{d} r_{1} \mathrm{~d} r_{2} \mathrm{~d} r_{3},
$$

where $\mathbf{B}$ is displacement-strain transformation matrix, and $\mathbf{D}_{(i e)}\left(r_{1}, r_{2}, r_{3}\right)$ is matrix derived from material properties; $r_{1}, r_{2}$, and $r_{3}$ are natural coordinate systems, respectively, and $\operatorname{det}|J|$ is a determinant of Jacobian matrix for transformation between natural and global coordinate systems. Note that all components of B and $\operatorname{det}|J|$ are constant in structured problems as basis functions are uniform. We use voxel elements that consists of eight smaller voxels of size $(d s / 2)^{3}$ and possesses constant isotropic linear elastic material properties. Therefore, the matrix $\mathbf{D}_{(i e)}\left(r_{1}, r_{2}, r_{3}\right)$ is constant in each subvoxel. We denote the matrix from the $i$-th of eight subvoxels in $i e$-th element as $\mathrm{D}_{(i e)}^{i}$. The matrix can be described as follows:

$$
\begin{aligned}
\mathbf{D}_{(i e)}^{i}=K_{(i e)}^{i}\left(\begin{array}{llllll}
1 & 1 & 1 & 0 & 0 & 0 \\
1 & 1 & 1 & 0 & 0 & 0 \\
1 & 1 & 1 & 0 & 0 & 0 \\
0 & 0 & 0 & 0 & 0 & 0 \\
0 & 0 & 0 & 0 & 0 & 0 \\
0 & 0 & 0 & 0 & 0 & 0
\end{array}\right) \\
+G_{(i e)}^{i}\left(\begin{array}{cccccc}
4 / 3 & -2 / 3 & -2 / 3 & 0 & 0 & 0 \\
-2 / 3 & 4 / 3 & -2 / 3 & 0 & 0 & 0 \\
-2 / 3 & -2 / 3 & 4 / 3 & 0 & 0 & 0 \\
0 & 0 & 0 & 1 & 0 & 0 \\
0 & 0 & 0 & 0 & 1 & 0 \\
0 & 0 & 0 & 0 & 0 & 1
\end{array}\right),
\end{aligned}
$$

where $K_{(i e)}^{i}$ and $G_{(i e)}^{i}$ are bulk modulus and shear modulus for the $i$-th of eight subvoxels in $i e$-th element.

Considering the matrices in the right side of Eq. (4) are also constant, the element stiffness matrix in Eq. (3) is represented as linear combination of two matrices and coefficients derived from $K_{(i e)}^{i}$ and $G_{(i e)}^{i}$. Eventually, the multiplication of the element stiffness matrix and element vector $\mathbf{u}_{(i e)}$ becomes

$$
\mathbf{f}_{(i e)}=\sum_{i=1}^{8}\left(\alpha_{i(i e)} \mathbf{P}_{i}+\beta_{i(i e)} \mathbf{Q}_{i}\right) \mathbf{u}_{(i e)} .
$$

Here, we redefine the scalar values corresponding to the material properties $K_{(i e)}^{i}$ and $G_{(i e)}^{i}$ as $\alpha_{i(i e)}$ and $\beta_{i(i e)}$. When we extend our method to visco-elastic analysis, we can apply the same procedure by setting appropriate coefficients $\alpha_{i(i e)}$ and $\beta_{i(i e)}$. $\mathbf{P}_{i}$ and $\mathbf{Q}_{i}$ denote the $24 \times 24$ matrices with constant values and $\mathbf{u}_{(i e)}$ is the $i e$-th element displacement data of size of 24 . The result of the 


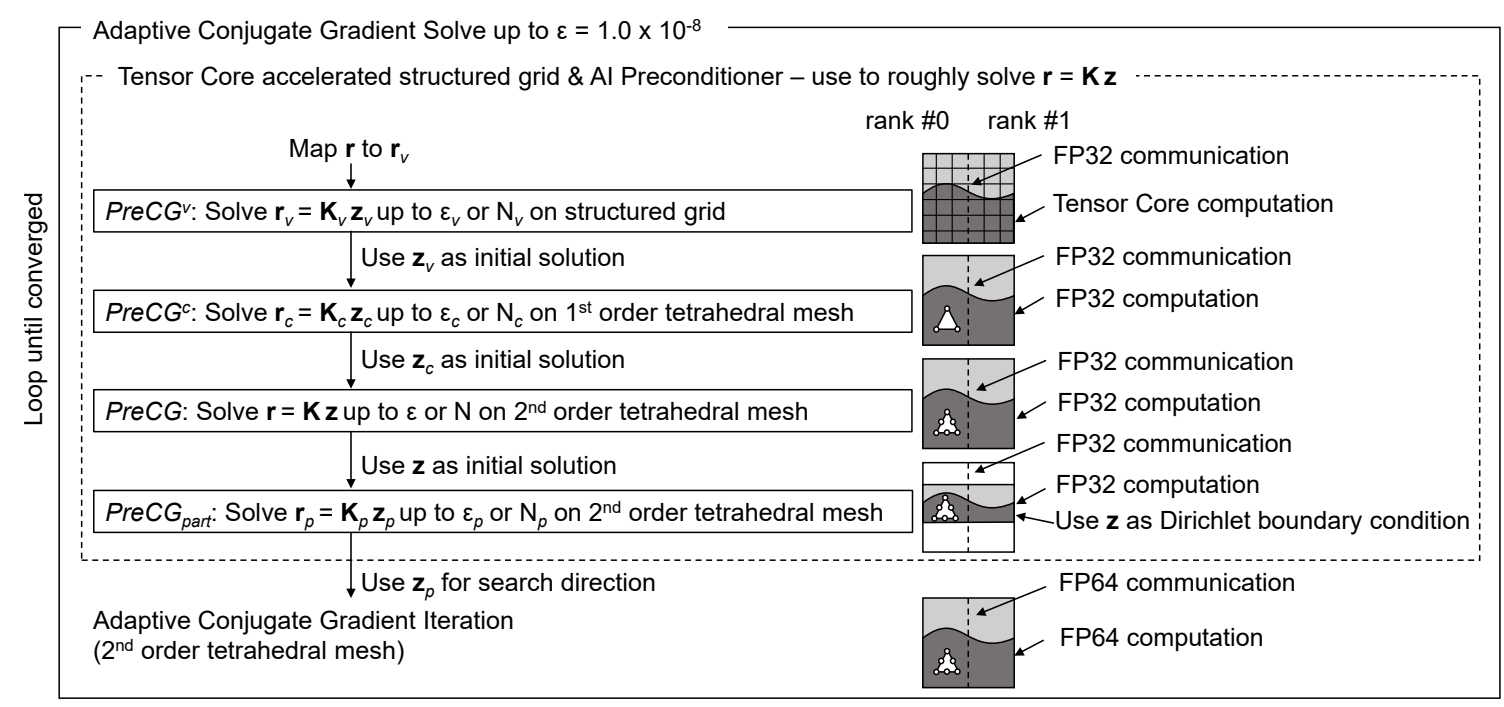

Figure 1: Solver algorithm. The linear set of the equations of the unstructured second-order tetrahedral mesh is computed in double precision using an adaptive conjugate gradient-based preconditioner, which consists of a Tensor Core-accelerated structured grid solver, a first-order tetrahedral element solver, and a second-order tetrahedral element solver. Point-to-point communication is overlapped with the computations following previous studies [31] so that it does not become a bottleneck of the performance.

matrix-vector product $\mathrm{f}_{(i e)}$ is added into the global left-hand side vector. Although we use an eight-node voxel element, it reflects the heterogeneous material properties of the eight subvoxels, and so, can be considered a special type of coarsening that reflects $c_{i j k l}$ with small data access costs. Considering that $\mathbf{P}_{i}$ and $\mathbf{Q}_{i}$ are constant among the elements, we can gather $\mathbf{u}_{(1)}, \mathbf{u}_{(2)}, \ldots$ to generate a matrix to conduct a matrix-matrix multiplication: $\mathbf{P}_{i}\left(\mathbf{u}_{(1)}, \mathbf{u}_{(2)}, \ldots\right)$ and $Q_{i}\left(\mathbf{u}_{(1)}, \mathbf{u}_{(2)}, \ldots\right)$. We use Tensor Core for this matrix-matrix multiplication part. Peak FLOP performance on Tensor Core is 4fold of that in FP16 computations on Volta GPUs. Therefore, it is faster to compute for each constant matrix $\mathbf{P}_{i}$ and $\mathbf{Q}_{i}$ as matrixmatrix multiplication on Tensor Core even though the number of required floating-point number operations increases.

Tensor Core is designed to accelerate computations requiring accuracies less than FP32; hence, special care is required to use these arithmetic units for the above computations. Compared to deep learning etc., the conjugate gradient method is prone to numerical errors; therefore, we design an algorithm that is stable even when using reduced precision data types. We add two methods such that the solver converges even when half-precision arithmetic is used by Tensor Core on a Volta GPU. First, we introduce local normalization. We rescale vector $\mathbf{u}_{(i e)}$ (originally in FP32) such that its values are within the range of FP16. After the computation, we reflect the scaling factors when adding $\mathbf{f}_{(i e)}$ in FP32. Secondly, we remove the rigid body motion from the input vectors to circumvent the rounding errors incurred in the computing process. This removal results in the convergence of the solver with an iteration count slightly higher than in FP32.

\section{PERFORMANCE TUNING FOR TENSOR CORE COMPUTATION}

We design an algorithm that computes much of the computation cost in Eq. (5) on Tensor Core, AI-specific hardware on a Volta GPU. On the other hand, to reduce the time-to-solution of the solver, we have to consider the computational characteristics required in our target computations, which are different to those of AI. Firstly, this kernel involves the read/write operations of the element vectors $\mathbf{p}_{(i e)}$ and $\mathbf{q}_{(i e)}$; hence, it may become memory bound. Second, the matrix size is much smaller than those used in typical deep learning applications and leads to relatively large memory access costs. Therefore, thorough reductions of the data access costs are essential for utilizing the potential of Tensor Core for this kernel. We explain below how we develop the algorithm to reduce access cost for global memory and shared memory.

\subsection{Reduction of the device memory access costs}

Reductions of the read/write costs of vectors $\mathbf{u}_{(i e)}$ and $\mathbf{f}_{(i e)}$ before and after Tensor Core's computations are required for higher performance. So, we introduce warp specialization and overlap the data movements with Tensor Core's computations. First, we decompose the domain allocated to each GPU into 80 subdomains, each for the 80 streaming multiprocessors of the V100 GPUs. One thread block synchronously computes one $x-y$ plane at a time and moves in the $z$ direction. As shown in Fig. 2, we separate the threads into two groups: the first group computes the matrix vector products by using the data in the shared memory and writes the results back to the shared memory, whereas the other group moves the data between the shared memory and the device memory. While 


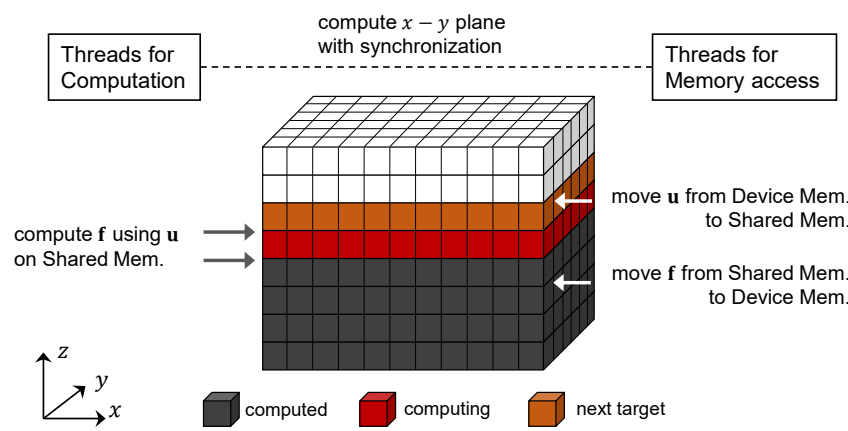

Figure 2: Rough image of warp specialization in the kernel.

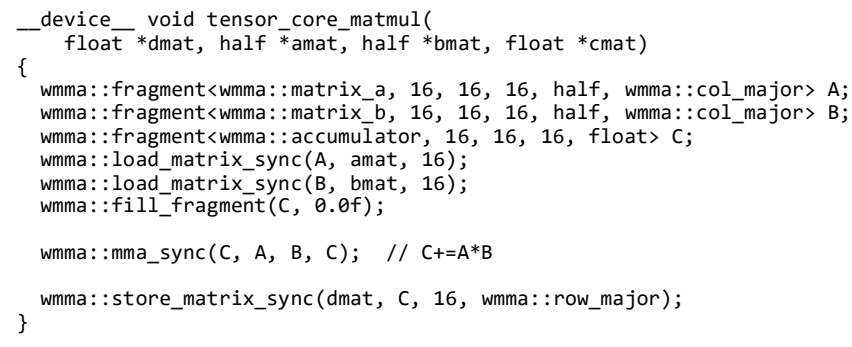

Figure 3: Example code for using Tensor Cores in CUDA C.

the computation group is computing the matrix-vector products of elements between $z=i$ and $z=i+1$, the data movement group adds the results of plane $z=i-1$ in the shared memory to the device memory via atomicAdd and reads the element vectors for plane $z=i+2$ to the shared memory. This procedure improves the utilization ratio of Tensor Core's computations.

\subsection{Efficient data mapping for high performance computations on small matrices}

We use the warp-level matrix multiply and accumulate (wmma) API, which is capable of low-level programming, to utilize Tensor Core's computations (Fig. 3). This API synchronously computes the products of $m \times k$ with $k \times n$ matrices using 32 threads. The current wmma API supports sizes $(m, n, k)=(16,16,16),(8,32,16),(32,8,16)$. The data must be moved from the device or shared memory to the registers via wmma :: load_matrix_sync. The data are computed by wmma :: mma_sync. Afterward, the results on the registers are stored to the device or shared memory by wmma :: store_matrix_sync.

To compute Eq. (5), Tensor Core's operations for the multiplication of $\mathbf{P}_{i}\left(\mathbf{u}_{(1)}, \mathbf{u}_{(2)}, \ldots\right)$ and $\mathbf{Q}_{i}\left(\mathbf{u}_{(1)}, \mathbf{u}_{(2)}, \ldots\right)$ must be issued for 32 threads. In contrast, our element-wise computations, including the scaling/rescaling of the element vectors $\mathbf{u}_{(i e)}$ and $\mathbf{f}_{(i e)}$, as well as the multiplication of the material properties $\alpha_{i(i e)}$ and $\beta_{i(i e)}$, must assign one thread to a specific element. These assignments lead to a large difference in the thread allocation between Tensor Core's computation part and the other parts. When we conduct the element-wise operations before or after Tensor Core's operations, each thread must retrieve the corresponding data via shared

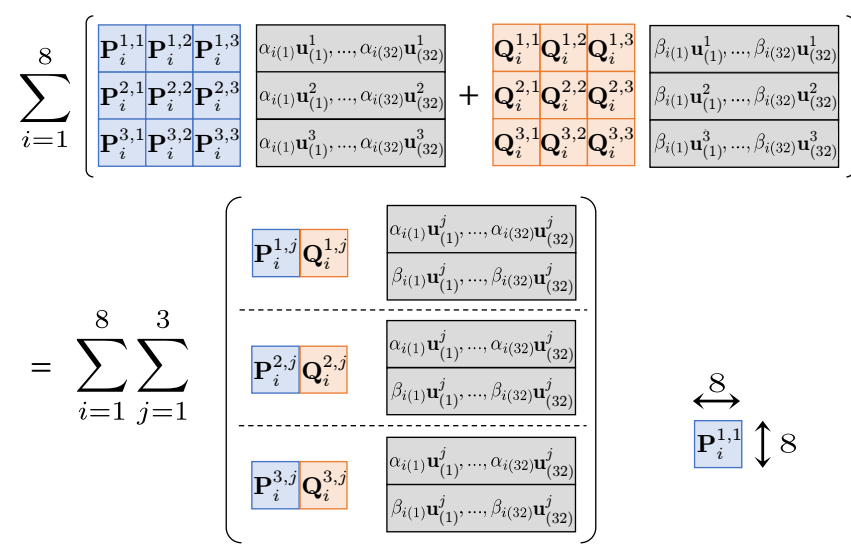

Figure 4: Decomposition of the matrices in the multiplication to compute $\sum_{i=1}^{8}\left(\mathbf{P}_{i}\left(\alpha_{i(i e)} \mathbf{u}_{(i e)}\right)+Q_{i}\left(\beta_{i(i e)} \mathbf{u}_{(i e)}\right)\right)$ on Tensor Core. Constant matrices have a size of $8 \times 16$ and the matrices composed of vectors have a size of $16 \times 32$.

(a) Memory alignment of the $8 \times 16$ matrix seen from users

\begin{tabular}{|c|c|c|c|c|c|c|c|c|c|c|c|c|c|c|c|}
\hline 0 & 1 & 2 & 3 & 4 & 5 & 6 & 7 & 8 & 9 & 10 & 11 & 12 & 13 & 14 & 15 \\
\hline 16 & 17 & 18 & 19 & 20 & 21 & 22 & 23 & 24 & 25 & 26 & 27 & 28 & 29 & 30 & 31 \\
\hline 32 & 33 & 34 & 35 & 36 & 37 & 38 & 39 & 40 & 41 & 42 & 43 & 44 & 45 & 46 & 47 \\
\hline 48 & 49 & 50 & 51 & 52 & 53 & 54 & 55 & 56 & 57 & 58 & 59 & 60 & 61 & 62 & 63 \\
\hline 64 & 65 & 66 & 67 & 68 & 69 & 70 & 71 & 72 & 73 & 74 & 75 & 76 & 77 & 78 & 79 \\
\hline 80 & 81 & 82 & 83 & 84 & 85 & 86 & 87 & 88 & 89 & 90 & 91 & 92 & 93 & 94 & 95 \\
\hline 96 & 97 & 98 & 99 & 100 & 101 & 102 & 103 & 104 & 105 & 106 & 107 & 108 & 109 & 110 & 111 \\
\hline 112 & 113 & 114 & 115 & 116 & 117 & 118 & 119 & 120 & 121 & 122 & 123 & 124 & 125 & 126 & 127 \\
\hline
\end{tabular}

wmma: : load_matrix_sync

(b) Register mapping of the $8 \times 16$ matrix for the Tensor Core computation

registers $0,1, \ldots, 15$

\begin{tabular}{|c|c|c|c|c|c|c|c|c|c|c|c|c|c|c|c|c|}
\hline$\%$ & & 1 & 2 & 3 & 32 & 33 & 34 & 35 & 64 & 65 & \begin{tabular}{|l|}
66 \\
\end{tabular} & 67 & 96 & 97 & 98 & 99 \\
\hline & & 9 & 10 & 11 & 40 & 41 & 42 & 43 & 72 & 73 & 74 & 75 & 104 & 105 & $\overline{06}$ & 107 \\
\hline & 16 & 17 & 18 & 19 & 48 & , & 50 & 51 & 80 & 81 & 82 & 33 & 11 & & & 115 \\
\hline & 24 & 25 & 26 & 27 & 56 & 57 & 58 & 59 & 88 & 89 & 90 & 91 & 120 & 121 & 16 & 123 \\
\hline$=16,20,24,28$ & 4 & 5 & 6 & 7 & 36 & 37 & 38 & 39 & 68 & 69 & 70 & 71 & 100 & 101 & 102 & 10 \\
\hline $2=17,21,25,29$ & 12 & 13 & 14 & 15 & 44 & 45 & 46 & 47 & 76 & 77 & 78 & 79 & 108 & 109 & 110 & 111 \\
\hline 18 & 20 & 21 & 22 & 23 & 52 & 53 & 54 & 55 & 84 & 85 & 86 & 87 & 116 & 11 & 11 & 119 \\
\hline 19,23 & 28 & 29 & 30 & 31 & 60 & 61 & 62 & 63 & 92 & 93 & 94 & 95 & & & & \\
\hline
\end{tabular}

Figure 5: Relationship of the data allocation in the wmma API. When we call wmma :: load_matrix_sync, each thread refers to a specific portion of the matrix according to its index and stores the data in the registers.

memory. We can call wmma API for this data remapping; however, doing so increases the amount of shared memory access. If we used Tensor Core for large matrices, we could decompose the matrices into many sub-matrices and reuse the same sub-matrix many times, thereby covering the costs of storing the results into the shared memory and remapping to the registers. However, our matrix is so small that the performance of the kernel easily becomes shared memory bandwidth bound when we request overly frequent remapping. Therefore, we minimize the number of data remapping 


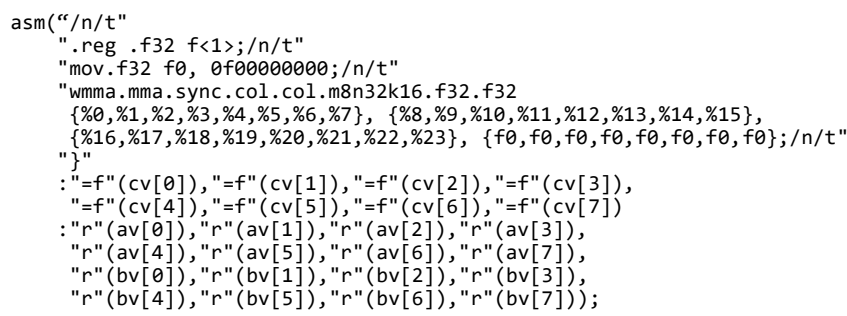

Figure 6: Example code for using Tensor Core with the PTX assembly in CUDA C. Note that two FP16 values, namely av and bv, are packed into an INT32 register; thus, only eight registers are designated in the assembly.

operations by the efficient decomposition of the matrices and by the direct input of the necessary data into the registers after decoding the data remapping in the wmma API. The details of this implementation are described below.

First, we modify our algorithm such that we can reuse the same matrix as many times as possible and obtain the results by the simple summation of the matrix-matrix multiplications (Fig. 4). We compute the multiplication on the order of $\sum_{i=1}^{8}\left(\mathbf{P}_{i}\left(\alpha_{i(i e)} \mathbf{u}_{(i e)}\right)+\mathbf{Q}_{i}\left(\beta_{i}\right.\right.$
The computations of the 32 elements are subdivided into 72 Tensor Core operations with the block size $(m, n, k)=(8,32,16)$. Here, sub-matrices $\mathrm{P}_{i}^{i x, i y}$ and $\mathrm{Q}_{i}^{i x, i y}$ with indexes $i x$ and $i y$ are of size $8 \times 8$ and satisfies

$$
\mathbf{P}_{i}=\left(\begin{array}{lll}
\mathbf{P}_{i}^{1,1} & \mathbf{P}_{i}^{1,2} & \mathbf{P}_{i}^{1,3} \\
\mathbf{P}_{i}^{2,1} & \mathbf{P}_{i}^{2,2} & \mathbf{P}_{i}^{2,3} \\
\mathbf{P}_{i}^{3,1} & \mathbf{P}_{i}^{3,2} & \mathbf{P}_{i}^{3,3}
\end{array}\right), \quad \mathbf{Q}_{i}=\left(\begin{array}{lll}
\mathbf{Q}_{i}^{1,1} & \mathbf{Q}_{i}^{1,2} & \mathbf{Q}_{i}^{1,3} \\
Q_{i}^{2,1} & \mathbf{Q}_{i}^{2,2} & \mathbf{Q}_{i}^{2,3} \\
Q_{i}^{3,1} & Q_{i}^{3,2} & Q_{i}^{3,3}
\end{array}\right),
$$

and vector $\mathbf{u}_{(i e)}^{i x}$ with index $i x$ is of size 8 and satisfies $\mathbf{u}_{(i e)}^{T}=$

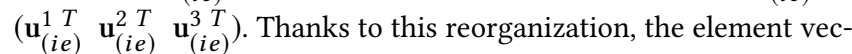
tor matrix of size $16 \times 32\left(\alpha_{i(i e)} \mathbf{u}_{(i e)}^{j}\right.$ and $\beta_{i(i e)} \mathbf{u}_{(i e)}^{j}$ in Fig. 4) can be reused several times. We can also compute by extracting the multiplication results in the shared memory just once per 32 elements with wmma :: store_matrix_sync, thereby significantly reducing the number of accesses to the shared memory.

Second, when we construct the matrices out of the element vectors $\left(\alpha_{i(i e))} \mathbf{u}_{(i e)}^{j}\right.$ and $\beta_{i(i e)} \mathbf{u}_{(i e)}^{j}$ in Fig. 4), we omit the redundant wmma :: load_matrix_sync calls. For example, in the wmma API of the current CUDA version, it is found that each thread uses 16 FP16 numbers, thereby leading to $16 \times 32=512$ values among the 32 threads with duplication among threads as shown in [38] (the 8 $\times 16$ matrix mapping is shown in Fig. 5). Using this property, we can call the wmma API from the PTX assembly (Fig. 6) and remove the data movement via shared memory, i.e., element-wise vectors $\alpha_{i(i e)} \mathbf{u}_{(i e)}^{j}$ and $\beta_{i(i e)} \mathbf{u}_{(i e)}^{j}$ can be always in the registers.

\section{PERFORMANCE MEASUREMENT}

\subsection{Problem settings}

We target a two-layered problem that mimicks the Earth's crust and mantle (Fig. 7). The interface of the layers has a wave-shaped geometry with sharp material discontinuity at the interface. To see the effects of transprecision computing in different problem settings,

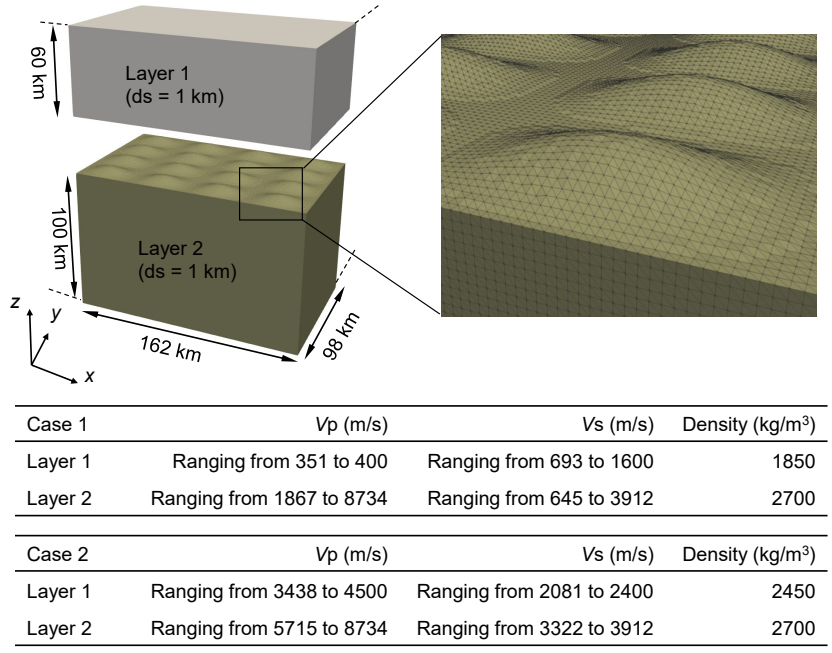

Figure 7: Measurement model consisting of the unstructured second-order tetrahedral elements of each GPU. The model is duplicated in the $x$ - and $y$-directions according to the number of compute nodes. $\left.\beta_{i(i e)} \mathbf{u}_{(i e)}\right)$.

we use two settings. We compute the displacement response to a given fault slip in Case 1 and the displacement response to gravity in Case 2. The material properties change in the depth directions inside the crust and the mantle; hence, the material within each layer of this model is changed as well. The bottom of the model is fixed. Figure 7 shows a model per GPU. We duplicate this model in the $x$ - and $y$-directions to create a model for 216 GPUs. The total degrees of freedom (DOF) are 13,257,281,664 and the DOFs in the preconditioning solvers PreCG, PreCG ${ }^{c}$, PreCG $^{v}$, and PreCG $G_{\text {part }}$ are $13,257,281,664,2,011,200,192,1,683,540,936$, and 472,350,744, respectively.

We use this problem to compare the performance of our proposed solver, the standard PCGE, and the SC14 solver. PCGE is a standard conjugate gradient solver with $3 \times 3$ block Jacobi conditioning [41] and an element-by-element [48] matrix-vector kernel tuned for the V100 architecture. The SC14 solver is a tuned version of the state-of-the-art solver in [21] and it is our proposed solver without PreCG ${ }^{v}$ and PreCG $G_{\text {part }}$ while PCGE is our proposed solver without PreCG${ }^{v}$, PreCG ${ }^{c}$, PreCG, and PreCG $G_{\text {part }}$. Here, all the major computations of the solver are ported and tuned for the V100 architecture. FP64 is used for the computation throughout the PCGE solver, whereas both FP64 and FP32 are used in SC14 solver (FP32 is used for the multi-grid preconditioner). To clarify the effect of the differences among the solver algorithms, all techniques to improve the performance of preconditioning solvers are also implemented on the SC14 solver and PCGE, including the reduction of the memory access costs in an element-by-element method by the use of the shared memory and the overlapping of point-to-point communications and matrix-vector multiplications. Namely, the difference in performance of solvers results from only whether each preconditioning solver $\left(\right.$ PreCG $^{v}$, PreCG $^{c}$, PreCG, or PreCG $\left.G_{\text {part }}\right)$ is enabled, and there is no difference in detailed implementations. Note that the relative error tolerances of all solvers are set to $\epsilon=1.0 \times 10^{-8}$. 
Table 1: Performance of kernel on one V100 GPU of Summit. Peak bandwidths are extracted from [26].

\begin{tabular}{|c|c|c|c|c|c|c|c|c|c|}
\hline & \multirow{2}{*}{$\begin{array}{l}\text { elapsed } \\
\text { time }\end{array}$} & \multirow[t]{2}{*}{ FLOPS } & \multicolumn{2}{|c|}{$\begin{array}{l}\text { device mem. } \\
\text { throughput }\end{array}$} & \multicolumn{2}{|c|}{$\begin{array}{l}\text { L2 cache } \\
\text { throughput }\end{array}$} & \multirow{2}{*}{$\begin{array}{c}\text { unified cache } \\
\text { throughput } \\
\text { load+store }\end{array}$} & \multicolumn{2}{|c|}{$\begin{array}{c}\text { shared mem. } \\
\text { throughput }\end{array}$} \\
\hline & & & read & write & read & write & & load & store \\
\hline FP32 & $3.830 \mathrm{~ms}$ & 12.1 TFLOPS & $62 \mathrm{~GB} / \mathrm{s}$ & $8.6 \mathrm{~GB} / \mathrm{s}$ & $189 \mathrm{~GB} / \mathrm{s}$ & $29 \mathrm{~GB} / \mathrm{s}$ & $11343 \mathrm{~GB} / \mathrm{s}$ & $11179 \mathrm{~GB} / \mathrm{s}$ & $86 \mathrm{~GB} / \mathrm{s}$ \\
\hline FP16 & $2.527 \mathrm{~ms}$ & 18.3 TFLOPS & $114 \mathrm{~GB} / \mathrm{s}$ & $29 \mathrm{~GB} / \mathrm{s}$ & $349 \mathrm{~GB} / \mathrm{s}$ & $77 \mathrm{~GB} / \mathrm{s}$ & $10227 \mathrm{~GB} / \mathrm{s}$ & $9934 \mathrm{~GB} / \mathrm{s}$ & $135 \mathrm{~GB} / \mathrm{s}$ \\
\hline typical TC & $3.327 \mathrm{~ms}$ & 13.9 TFLOPS & $76 \mathrm{~GB} / \mathrm{s}$ & $14 \mathrm{~GB} / \mathrm{s}$ & $424 \mathrm{~GB} / \mathrm{s}$ & $61 \mathrm{~GB} / \mathrm{s}$ & $3267 \mathrm{~GB} / \mathrm{s}$ & $1498 \mathrm{~GB} / \mathrm{s}$ & $9323 \mathrm{~GB} / \mathrm{s}$ \\
\hline tuned TC & $0.930 \mathrm{~ms}$ & 49.7 TFLOPS & $264 \mathrm{~GB} / \mathrm{s}$ & $37 \mathrm{~GB} / \mathrm{s}$ & $541 \mathrm{~GB} / \mathrm{s}$ & $123 \mathrm{~GB} / \mathrm{s}$ & $8155 \mathrm{~GB} / \mathrm{s}$ & $7435 \mathrm{~GB} / \mathrm{s}$ & $905 \mathrm{~GB} / \mathrm{s}$ \\
\hline \multicolumn{3}{|c|}{ peak bandwidths } & \multicolumn{2}{|c|}{$750 \mathrm{~GB} / \mathrm{s}$} & \multicolumn{2}{|c|}{$2155 \mathrm{~GB} / \mathrm{s}$} & $12080 \mathrm{~GB} / \mathrm{s}$ & \multicolumn{2}{|c|}{$12080 \mathrm{~GB} / \mathrm{s}$} \\
\hline
\end{tabular}

For our proposed solver, we use $0.01,0.08,0.1$, and $1.0 \times 10^{-3}$ as the error tolerances of the PreCG ${ }^{v}$, PreCG $^{c}$, PreCG, and PreCG $G_{\text {part }}$ preconditioning solvers, respectively. Also, we use 2000, 50, 50, and 50 as the maximum numbers of iterations in the PreCG ${ }^{v}, \operatorname{PreCG}^{c}$, PreCG, and PreCG $G_{\text {part }}$ solvers, respectively. We compare the performances of the solvers on Summit [44]. We use herein one GPU per MPI process (six MPI processes per node) and MPI_W time to measure the elapsed time. nvprof is used to measure FP32 and FP64 FLOPS while Tensor Core's instructions are counted from the code. All the measurements include outputting the displacement results on the ground surface to the file system. All measurements were confirmed to have the same double-precision results regardless of the method used (our proposed solver, SC14 solver, and PCGE) within a relative error tolerance of $10^{-8}$.

\subsection{Computation environment}

Summit comprises 4608 IBM Power System AC922 nodes, each with two IBM POWER9 (22 SIMD multi-core) processors and six NVIDIA Tesla V100 [34] accelerators. Each node has 512 GB of DDR4 memory for use by the POWER9 processors. Each of the six accelerators has $16 \mathrm{~GB}$ of $900 \mathrm{~GB} / \mathrm{s}$ high bandwidth memory (HBM2). The peak FP64 performance of a node is 7.8 TFLOPS $\times 6=46.8$ TFLOPS, which yields a total of 215 PFLOPS for the entire system. The FP32 peak performance is double that of FP64, while the FP16 peak performance is quadruple that of FP64 when using doublewidth arithmetic units. Tensor Cores with mixed FP16 and FP32 arithmetic are also available and lead to 125 TFLOPS per GPU. The compute nodes are connected to a dual-rail EDR InfiniBand network with a node injection bandwidth of $25 \mathrm{~GB} / \mathrm{s}$ in both send/receive directions. GPUDirect RDMA [13] is available for circumventing memory copies between the device memory and the host memory during MPI communications. We used CUDA version 10.1.168 and PGI compiler version 19.7 for performance measurement.

\subsection{Performance of the structured grid matrix-vector product kernel}

Firstly, we evaluated the performance of the matrix-vector kernel in the structured problem by using one Volta GPU on Summit. We targeted the inner domain of Fig. 7. That is, we computed multiplication for all elements except those on the outer faces of the domain, which accounted for the largest proportion of the computation costs in the PreCG ${ }^{v}$ solver and was required for the overlapping of the point-to-point communications and matrix-vector multiplications when multiple GPUs were used. The number of elements to compute was $2,457,000$. Here, we compared four versions in total.
First, one version computed the multiplication in single precision, whereas the second version computed in half precision. Hereinafter, these versions are referred to as FP32 kernel and FP16 kernel, respectively. Regarding Tensor Core-enhanced kernels, we used a typical TC kernel and a tuned TC kernel. The typical TC kernel always called wmma API to map the components of the matrices between the shared memory and registers while the tuned TC kernel reduced the access costs to shared memory by explicit mapping with PTX assembly. All these kernels had the same access patterns to vectors $\mathbf{u}_{(i e)}$ and $\mathbf{f}_{(i e)}$; thus, the access patterns to the device memory were the same. We decomposed the $24 \times 24$ matrices into $8 \times 8$ sub-matrices for all the kernels as was the case with the tuned TC kernel. Except for the FP32 kernel, we normalized each element vector and removed the rigid body motion to circumvent numerical errors. The elapsed time, FLOPS, and measured memory bandwidth are summarized in Table 1.

With the FP32 kernel, we attained $77.6 \%$ of peak FLOPS in single precision; thus, we confirmed that our target kernel had achieved dense computation. Regarding the measured bandwidths, those of the shared memory and unified cache were close to the peak bandwidth noted in [26]. Compared to these values in the FP32 kernel, the FP16 kernel reduced the footprints of the shared memory and unified cache by using half precision instead of single precision for the matrix components. The FP16 kernel attained a 1.52-fold speedup over the FP32 kernel. This ratio was lower than that of the peak FLOPS between single and half precision on a Volta GPU. The normalization of the element vectors and data conversion were considered as possible reasons. As for the kernels using Tensor Core, we confirmed via a comparison between the typical TC kernel and tuned TC kernel that the costs of data access to shared memory had a large effect on the performance. High bandwidth in shared memory was measured in both kernels and was assumed to be the bottleneck of the performance. These kernels had lower bandwidths of the unified cache than did the other kernels because each thread in FP32 kernel and FP16 kernel had to access all the components of the $24 \times 24$ matrices, whereas each thread in the typical TC kernel and tuned TC kernel had access only to portions of the matrices. The typical TC kernel frequently used shared memory for mapping between the registers and shared memory; thus, much storage to shared memory was required and the elapsed time was longer than for the FP16 kernel. In contrast, the tuned TC kernel greatly reduced the access cost to shared memory and relieved the bottleneck. The tuned TC kernel attained 49.7 TFLOPS, which was high as compared to the performance for typical matrix-matrix multiplication. For example, [29] reported that a batched GEMM using $16 \times 16$ matrices had achieved 5 TFLOPS on a Volta GPU. When we compared the 
Table 2: Number of iteration in each solvers. Proposed solver uses four types of data types in matrix-vector multiplication part of PreCG $^{v}$ solver.

\begin{tabular}{l|ccccc}
\multicolumn{1}{c|}{$\begin{array}{c}\text { Case 1 } \\
\text { solver }\end{array}$} & the number of iterations \\
\hline PCGE & 5226 & - & - & - & - \\
SC14 & 10 & 219 & 2412 & - & - \\
prop. (FP32) & 8 & 103 & 108 & 1917 & 154 \\
prop. (FP16) & 8 & 102 & 108 & 1920 & 154 \\
prop. (tuned TC) & 8 & 102 & 108 & 1920 & 154 \\
prop. (bfloat16) & 8 & 102 & 105 & 1876 & 172 \\
\hline & \multicolumn{5}{|c}{} \\
Case 2 & \multicolumn{5}{|c}{ the number of iterations } \\
solver & $C G$ & PreCG & PreCG & PreCG & PreCG $G_{\text {part }}$ \\
\hline PCGE & 3520 & - & - & - & - \\
SC14 & 8 & 124 & 4086 & - & - \\
prop. (FP32) & 8 & 104 & 143 & 2604 & 47 \\
prop. (FP16) & 8 & 98 & 196 & 2721 & 97 \\
prop. (tuned TC) & 8 & 96 & 186 & 2448 & 100 \\
prop. (bfloat16) & 8 & 112 & 162 & 2720 & 45 \\
\hline
\end{tabular}

elapsed time of each kernel, the tuned TC kernel exhibited a 4.1-fold speedup over the FP32 kernel and a 2.7-fold speedup over the FP16 kernel.

\subsection{Convergence of the solver}

We used 36 compute nodes of Summit (216 GPUs) and evaluated the convergence and time-to-solution of the entire solver. In this subsection, we focused on the convergence of the solver. Here we additionally checked the convergence when using bfloat16, which is expected to be supported on some future architecture. This data type was not supported by a Volta GPU on hardware, so we emulated the convergence with the following procedures. First, we used the FP32 kernel with our proposed algorithm. The bfloat16 data type corresponded to a single-precision data type whose fraction part was truncated to seven bits; thus, we removed the lower 16 bits in the fraction parts of the single-precision numbers. When we used this data type, normalization of element vector $\mathbf{u}_{(i e)}$ and removal of the rigid body motion were applied to reduce numerical errors. These bit operations were conducted to $\mathbf{u}_{(i e)}$ before multiplication and $\mathbf{f}_{(i e)}$ after multiplication, respectively. In our problem settings, all the solvers converged in both Case 1 and Case 2 . The number of iterations in each solver did not significantly change even when we used the lower-precision data types including bfloat16 as shown in Table 2. Therefore, the effectiveness of using lower-precision data types by our proposed method was demonstrated.For comparison, we also measured the convergence when we used the proposed solver without PreCG ${ }^{v}$ solver. We used tuned TC kernel for matrix-vector kernel in $i v$. The number of iterations was $(C G$, PreCG, PreCG ${ }^{c}$, PreCG $\left._{\text {part }}\right)=(8,127,2572,278)$ in Case 1 and $(C G$, PreCG, PreCG $\left.{ }^{c}, \operatorname{Pre} C G_{\text {part }}\right)=(8,123,3901,110)$ in Case 2; therefore, we can see that the introduction of $\operatorname{PreCG}^{v}$ facilitated reducing the number of iteration especially in $\operatorname{PreCG}^{c}$.

We confirmed that the usage of lower-precision data types in the matrix-vector kernel had different effects on the convergence of the solver for Case 1 and Case 2. Its effect for the computation depends on the problem settings; therefore, we should choose the appropriate precision by considering arithmetic performance and convergence of the solver. In addition, the tolerance and the maximum number of the iterations in each preconditioning solver would have significant impacts on the entire computation cost. By optimizing these parameters, we can expect further performance improvement. This approach, including automatic tuning, would be one of our future tasks.

\subsection{Time to solution of the solver}

We compared the performance of our proposed solver to those of previous solvers. In addition to the tuned TC kernel, we measured the performance of the solver with the FP32 kernel and FP16 kernel. The FLOP counts and elapsed times of the entire solver are shown in Fig. 8. Compared to PCGE, the SC14 solver reduced the FLOP count by using adaptive preconditioning and the multi-grid method. The PreCG ${ }^{c}$ solver accounted for the largest proportion of the whole computation cost; thus, most computations were done in single precision, which led to further speedup than a decrease in the FLOP count. When we applied our proposed algorithm with the FP32 kernel, the total FLOP count was higher than that of the SC14 solver because the number of computations in the structured mesh was much higher. However, the rearrangement to a structured problem avoided the complex connectivity of the unstructured mesh and reduced the memory access costs, leading to dense computation and the high effective performance of the matrix-vector kernel; therefore, the time-to-solution did not increase as much as did the FLOP count. In fact, our proposed solver with the FP32 kernel required 6.8-fold and 7.9-fold FLOP count for Case 1 and Case 2 over the SC14 solver, but the time-to-solution was just 1.03 times and 1.10 times longer for each case. Much of the time-to-solution of the solver was spent on the PreCG ${ }^{v}$ part. For instance, matrix-matrix multiplication in $\operatorname{PreCG}^{v}$ in Case 1 took $7.43 \mathrm{~s}$, which accounted for $60 \%$ of the time-to-solution in the entire solver. Therefore, by switching the matrix-vector kernel from the FP32 kernel to the faster FP16 kernel or tuned TC kernel, we were able to improve the performance. We confirmed that the FLOP count did not change significantly with the FP16 kernel or tuned TC kernel, instead of the FP32 kernel. By using the tuned TC kernel for matrix-vector multiplication, we achieved a 1.7-fold speedup in both Case 1 and Case 2 in terms of time-to-solution as compared to the SC14 solver. The FLOPS in the entire solver reached 24.4 PFLOP/8.07 s/216 GPU $=14.0$ TFLOPS per GPU in Case 2 . When we skipped PreCG ${ }^{v}$ for comparison, the elapsed time of the solver with tuned TC kernel was $10.20 \mathrm{~s}$ in Case 1 and $13.24 \mathrm{~s}$ in Case 2, respectively. Judging from these results, we confirmed that the introduction of PreCG ${ }^{v}$ was a major factor in performance improvement. In summary, our proposed method required a much higher FLOP count than did previous solvers; however, the time-to-solution was shorter than that of the state-of-the-art SC14 solver because the matrix-matrix multiplication in our solver was rapidly computed by Tensor Core. Also, the speedup is insignificant when we did not compute on Tensor Core. Thus, we confirmed effectiveness of our proposed algorithm using AI-specific hardware. 
(a) Case 1
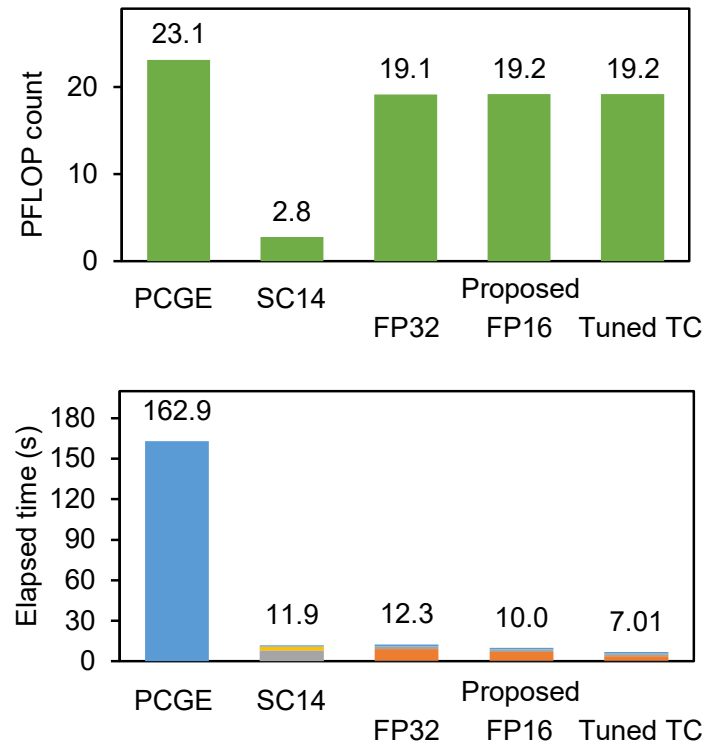

(b) Case 2
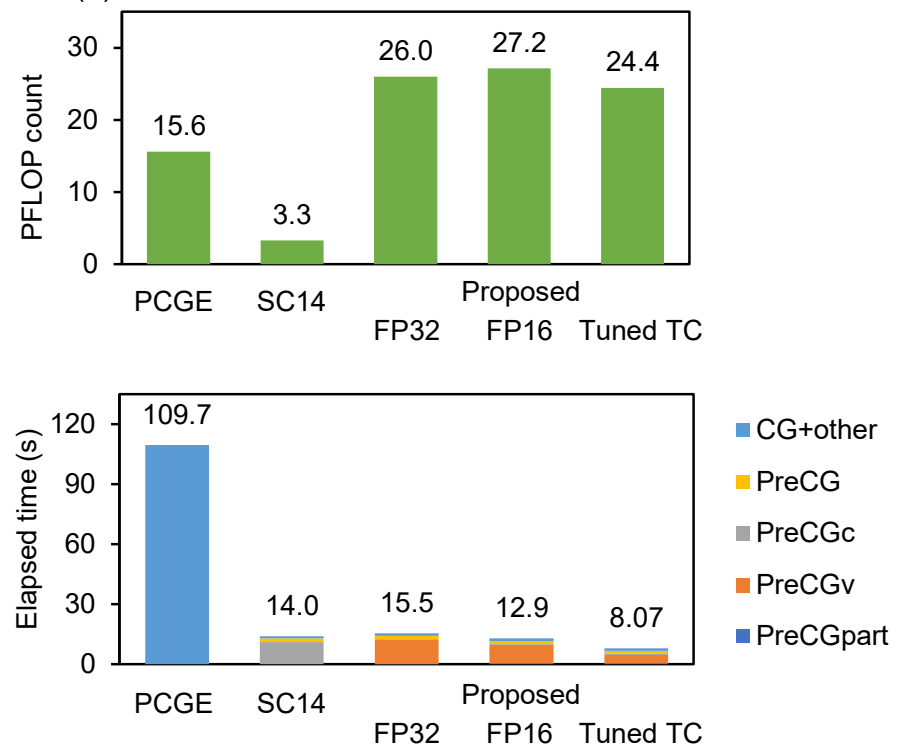

Figure 8: FLOP count and time-to-solution of the solvers.

\section{CONCLUSION}

The demand for crustal deformation computation methods using high-fidelity models has increased. Especially, methods to consider heterogeneous crustal structure (e.g., finite element modeling) are required. The combination of these crustal deformation computations and observed crustal deformation data will lead to a better understanding of earthquake processes. However, massive computation cost is required because the analyses target large domain with high resolution. In this study, we focused on AI-specific hardware and designed a low-order finite element solver that used Tensor Core on a Volta GPU for fast crustal deformation computations. We rearranged the solver algorithm so that most computations were executed as matrix-matrix multiplication in half precision. The sizes of our matrices in multiplication were smaller than those in typical applications of AI; thus, memory access costs stood out. However, by the optimization of matrix decomposition and thread parallelization, we obtained highly effective performance from Tensor Core even when we used $24 \times 24$ matrices. Our proposed solver required a much higher FLOP count than did previous solvers, but the timeto-solution was shorter as Tensor Core exhibited extremely high effective performance. Further experiments with more complex geometry would be required as future work.

Our proposed solver has been used in actual problems, such as those presented in [23]. The utility of our solver algorithm is expected to have broad impacts, such as the improvement of unstructured finite element solvers based on multi-grid methods. Furthermore, the highly accelerated $P r e C G^{v}$ solver could be used for voxel finite element methods [1] that are computationally costly analyses typically used in the design optimizations of industrial products.

\section{ACKNOWLEDGMENTS}

Our results were obtained using the Summit at Oak Ridge Leadership Computing Facility, a US Department of Energy, Office of Science User Facility at Oak Ridge National Laboratory (ORNL). We thank Yukihiko Hirano (NVIDIA) for coordination of the collaborative research project. We thank Christopher B. Fuson, Don E. Maxwell, Oscar Hernandez, Scott Atchley, Veronica MelesseVergara (ORNL), Jeff Larkin, Stephen Abbott (NVIDIA), Lixiang Luo (IBM), Richard Graham (Mellanox Technologies) for generous support concerning use of Summit. We thank Noda Tomoyuki and Hikaru Inoue (Fujitsu Limited) for support in program development. We acknowledge support from Japan Society for the Promotion of Science (18H05239 and 18K18873).

\section{REFERENCES}

[1] Niels Aage, Erik Andreassen, Boyan S Lazarov, and Ole Sigmund. 2017. Giga-voxel computational morphogenesis for structural design. Nature 550, 7674 (2017), 84.

[2] Ahmad Abdelfattah, Marc Baboulin, Veselin Dobrev, Jack Dongarra, Christopher Earl, Joel Falcou, Azzam Haidar, Ian Karlin, Tz Kolev, Ian Masliah, et al. 2016. High-performance tensor contractions for GPUs. Procedia Computer Science 80 (2016), 108-118.

[3] Ahmad Abdelfattah, Stanimire Tomov, and Jack Dongarra. 2019. Fast Batched Matrix Multiplication for Small Sizes using Half-Precision Arithmetic on GPUs. In 2019 IEEE International Parallel and Distributed Processing Symposium (IPDPS). IEEE, 111-122.

[4] Analog Computation in Flash Memory for Datacenter-scale AI Inference in a Small Chip, [Online]. 2018. https://www.hotchips.org/hc30/2conf2.05_Mythic Mythic_Hot_Chips_2018_V5.pdf.

[5] Arm's First-Generation Machine Learning Processor, [Online]. 2018. https://www.hotchips.org/hc30/2conf2.07_ARM_ML_Processor_HC30_ ARM_2018_08_17.pdf.

[6] Japan Hydrographic Association. 2013. JTOPO30 (30-second grid water depth data in Japan's coastal waters) [Online]. http://www.mirc.jha.jp/products/ finished/JTOPO30/.

[7] Erin Carson and Nicholas J Higham. 2018. Accelerating the solution of linear systems by iterative refinement in three precisions. SIAM fournal on Scientific Computing 40, 2 (2018), A817-A847. 
[8] Michael A Clark, Ronald Babich, Kipton Barros, Richard C Brower, and Claudio Rebbi. 2010. Solving Lattice QCD systems of equations using mixed precision solvers on GPUs. Computer Physics Communications 181, 9 (2010), 1517-1528.

[9] Kohei Fujita, Tsuyoshi Ichimura, Kentaro Koyama, Hikaru Inoue, Muneo Hori, and Lalith Maddegedara. 2017. Fast and Scalable Low-Order Implicit Unstructured Finite-Element Solver for Earth's Crust Deformation Problem. In Proceedings of the Platform for Advanced Scientific Computing Conference. ACM, 11.

[10] Yukitoshi Fukahata and Mitsuhiro Matsu'ura. 2005. General expressions for internal deformation fields due to a dislocation source in a multilayered elastic half-space. Geophysical fournal International 161, 2 (2005), 507-521.

[11] Gene H Golub and Qiang Ye. 1999. Inexact preconditioned conjugate gradient method with inner-outer iteration. SIAM Journal on Scientific Computing 21, 4 (1999), 1305-1320

[12] Google Announces Cloud TPU v2 Beta Availability for Google Cloud Platform, [Online]. 2018. https://www.anandtech.com/show/12429/google-cloudannounces-cloud-tpu-beta-availability.

[13] GPUDirect, [Online]. 2019. https://developer.nvidia.com/gpudirect.

14] Suyog Gupta, Ankur Agrawal, Kailash Gopalakrishnan, and Pritish Narayanan 2015. Deep learning with limited numerical precision. In International Conference on Machine Learning. 1737-1746.

[15] Azzam Haidar, Ahmad Abdelfattah, Mawussi Zounon, Panruo Wu, Srikara Pranesh, Stanimire Tomov, and Jack Dongarra. 2018. The design of fast and energy-efficient linear solvers: On the potential of half-precision arithmetic and iterative refinement techniques. In International Conference on Computational Science. Springer, 586-600.

[16] Azzam Haidar, Stanimire Tomov, Jack Dongarra, and Nicholas J Higham. 2018. Harnessing GPU tensor cores for fast FP16 arithmetic to speed up mixed-precision iterative refinement solvers. In SC18: International Conference for High Performance Computing, Networking, Storage and Analysis. IEEE, 603-613.

[17] Chihiro Hashimoto, Kenji Fukui, and Mitsuhiro Matsu'Ura. 2004. 3-D modelling of plate interfaces and numerical simulation of long-term crustal deformation in and around Japan. Pure and Applied Geophysics 161, 9-10 (2004), 2053-2068.

[18] Kristin LH Hughes, Timothy Masterlark, and Walter D Mooney. 2010. Poroelastic stress-triggering of the 2005 M8. 7 Nias earthquake by the 2004 M9. 2 SumatraAndaman earthquake. Earth and Planetary Science Letters 293, 3-4 (2010), 289-299.

[19] Thomas JR Hughes. 2012. The finite element method: linear static and dynamic finite element analysis. Courier Corporation.

[20] Tsuyoshi Ichimura, Kohei Fujita, Pher Errol Balde Quinay, Lalith Maddegedara, Muneo Hori, Seizo Tanaka, Yoshihisa Shizawa, Hiroshi Kobayashi, and Kazuo Minami. 2015. Implicit nonlinear wave simulation with $1.08 \mathrm{~T}$ DOF and $0.270 \mathrm{~T}$ unstructured finite elements to enhance comprehensive earthquake simulation. In SC'15: Proceedings of the International Conference for High Performance Computing, Networking, Storage and Analysis. IEEE, 1-12.

[21] Tsuyoshi Ichimura, Kohei Fujita, Seizo Tanaka, Muneo Hori, Maddegedara Lalith, Yoshihisa Shizawa, and Hiroshi Kobayashi. 2014. Physics-based urban earthquake simulation enhanced by $10.7 \mathrm{BlnDOF} \times 30 \mathrm{~K}$ time-step unstructured FE non-linear seismic wave simulation. In SC'14: Proceedings of the International Conference for High Performance Computing, Networking, Storage and Analysis. IEEE, 15-26.

[22] Tsuyoshi Ichimura, Kohei Fujita, Takuma Yamaguchi, Akira Naruse, Jack C Wells, Thomas C Schulthess, Tjerk P Straatsma, Christopher J Zimmer, Maxime Martinasso, Kengo Nakajima, et al. 2018. A fast scalable implicit solver for nonlinear time-evolution earthquake city problem on low-ordered unstructured finite elements with artificial intelligence and transprecision computing. In SC18 International Conference for High Performance Computing, Networking, Storage and Analysis. IEEE, 627-637.

[23] Tsuyoshi Ichimura, Kohei Fujita, Takuma Yamaguchi, Akira Naruse, Jack C Wells, Christopher J. Zimmer, Tjerk P. Straatsma, Takane Hori, Simone Puel, Thorsten W. Becker, Muneo Hori, and Naonori Ueda. 2019. 416-PFLOPS fast scalable implicit solver on low-ordered unstructured finite elements accelerated by 1.10-ExaFLOPS kernel with reformulated AI-like algorithm: For equationbased earthquake modeling. Research Poster for SC19: International Conference for High Performance Computing, Networking, Storage and Analysis (2019).

[24] Tsuyoshi Ichimura, Muneo Hori, and Hiroyuki Kuwamoto. 2007. Earthquake motion simulation with multiscale finite-element analysis on hybrid grid. Bulletin of the Seismological Society of America 97, 4 (2007), 1133-1143.

[25] Chetan Jhurani and Paul Mullowney. 2015. A GEMM interface and implementation on NVIDIA GPUs for multiple small matrices. F. Parallel and Distrib. Comput. 75 (2015), 133-140.

[26] Zhe Jia, Marco Maggioni, Benjamin Staiger, and Daniele P Scarpazza. 2018. Dissecting the NVIDIA Volta GPU architecture via microbenchmarking. arXiv preprint arXiv:1804.06826 (2018).

[27] William Kahan. 1996. IEEE standard 754 for binary floating-point arithmetic Lecture Notes on the Status of IEEE 754, 94720-1776 (1996), 11.

[28] C Kyriakopoulos, T Masterlark, S Stramondo, M Chini, and C Bignami. 2013. Coseismic slip distribution for the Mw 92011 Tohoku-Oki earthquake derived from 3-D FE modeling. Journal of Geophysical Research: Solid Earth 118, 7 (2013), 3837-3847.
[29] Stefano Markidis, Steven Wei Der Chien, Erwin Laure, Ivy Bo Peng, and Jeffrey S Vetter. 2018. NVIDIA Tensor Core programmability, performance \& precision. In 2018 IEEE International Parallel and Distributed Processing Symposium Workshops (IPDPSW). IEEE, 522-531.

[30] Timothy Masterlark. 2003. Finite element model predictions of static deformation from dislocation sources in a subduction zone: Sensitivities to homogeneous, isotropic, Poisson-solid, and half-space assumptions. Fournal of Geophysical Research: Solid Earth 108, B11 (2003).

[31] Paulius Micikevicius. 2009. 3D finite difference computation on GPUs using CUDA. In Proceedings of 2nd workshop on general purpose processing on graphics processing units. ACM, 79-84.

[32] SE Minson, M Simons, and JL Beck. 2013. Bayesian inversion for finite fault earthquake source models I-Theory and algorithm. Geophysical fournal International 194, 3 (2013), 1701-1726.

[33] NVIDIA. 2008. cuBLAS library. NVIDIA Corporation, Santa Clara, California 15, 27 (2008), 31

[34] NVIDIA. 2017. NVIDIA Tesla V100 GPU Architecture, [Online]. http://images. nvidia.com/content/volta-architecture/pdf/volta-architecture-whitepaper.pdf.

[35] Geospatial Information Authority of Japan. 2010. GNSS earth observation network system [Online]. http://terras.gsi.go.jp/geo_info/geonet_top.html.

[36] Yoshimitsu Okada. 1985. Surface deformation due to shear and tensile faults in a half-space. Bulletin of the seismological society of America 75, 4 (1985), 1135-1154.

[37] Jay Parker, Gregory Lyzenga, Charles Norton, Cinzia Zuffada, Margaret Glasscoe, John Lou, and Andrea Donnellan. 2008. Geophysical Finite-Element Simulation Tool (GeoFEST): algorithms and validation for quasistatic regional faulted crust problems. Pure and Applied Geophysics 165, 3-4 (2008), 497-521.

[38] Md Aamir Raihan, Negar Goli, and Tor M Aamodt. 2019. Modeling Deep Learning Accelerator Enabled GPUs. In 2019 IEEE International Symposium on Performance Analysis of Systems and Software (ISPASS). IEEE, 79-92.

[39] Johann Rudi, A Cristiano I Malossi, Tobin Isaac, Georg Stadler, Michael Gurnis, Peter WJ Staar, Yves Ineichen, Costas Bekas, Alessandro Curioni, and Omar Ghattas. 2015. An extreme-scale implicit solver for complex PDEs: highly heterogeneous flow in earth's mantle. In Proceedings of the international conference for high performance computing, networking, storage and analysis. 1-12.

[40] Youcef Saad. 1993. A flexible inner-outer preconditioned GMRES algorithm. SIAM fournal on Scientific Computing 14, 2 (1993), 461-469.

[41] Yousef Saad. 2003. Iterative methods for sparse linear systems. Vol. 82. siam.

[42] Hoo-Chang Shin, Holger R Roth, Mingchen Gao, Le Lu, Ziyue Xu, Isabella Nogues, Jianhua Yao, Daniel Mollura, and Ronald M Summers. 2016. Deep convolutional neural networks for computer-aided detection: CNN architectures, dataset characteristics and transfer learning. IEEE transactions on medical imaging 35, 5 (2016), 1285-1298.

[43] Japan Seismic Hazard Information Station. 2010. National Research Institute for Earth Science and Disaster Resilience [Online]. https://www.j-shis.bosai.go.jp/ download.

[44] Summit, [Online]. 2018. https://www.olcf.ornl.gov/olcf-resources/computesystems/summit/.

[45] Stanimire Tomov, Jack Dongarra, and Marc Baboulin. 2010. Towards dense linear algebra for hybrid GPU accelerated manycore systems. Parallel Comput. 36, 5-6 (2010), 232-240.

[46] Using bfloat16 with tensorflow models, [Online]. 2019. https://cloud.google.com/ tpu/docs/bfloat16.

[47] Kang Wang and Yuri Fialko. 2018. Observations and modeling of coseismic and postseismic deformation due to the $2015 \mathrm{Mw} 7.8$ Gorkha (Nepal) earthquake. fournal of Geophysical Research: Solid Earth 123, 1 (2018), 761-779.

[48] James M Winget and Thomas JR Hughes. 1985. Solution algorithms for nonlinear transient heat conduction analysis employing element-by-element iterative strategies. Computer Methods in Applied Mechanics and Engineering 52, 1-3 (1985), 711-815.

[49] Sencer Nuri Yeralan, Timothy A Davis, Wissam M Sid-Lakhdar, and Sanjay Ranka. 2017. Algorithm 980: Sparse QR factorization on the GPU. ACM Transactions on Mathematical Software (TOMS) 44, 2 (2017), 17. 